\title{
Pharyngeal Perforation after Anterior Cervical Fusion Surgery
}

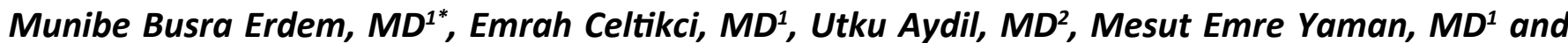 \\ Hakan Emmez, MD'
}

\author{
${ }^{1}$ Department of Neurosurgery, Gazi University Faculty of Medicine, Ankara, Turkey \\ ${ }^{2}$ Department of Otolaryngology - Head \& Neck Surgery, Gazi University Faculty of Medicine, Ankara, \\ Turkey
}

*Corresponding author: Munibe Busra Erdem, MD, Gazi University Faculty of Medicine, Department of Neurosurgery, Besevler, 06500, Ankara, Turkey, Orcid ID: 0000-0003-0373-8679, Tel: 00-90-536-286-3335, Fax: 00-90-312-212-9004

\begin{abstract}
Study design: A case report to emphasize the treatment of two different pharyngeal perforation cases, one of which occurs in early and one in the late post-operative period.

Objective: We aimed to remind the risk of pharyngeal perforation which is a rare but life-threatening complication in anterior cervical fusion surgery.

Summary and background data: Pharyngeal perforation is a serious complication of anterior cervical fusion surgery. If it is not diagnosed in the early period and the treatment is delayed, catastrophic consequences may occur which may cause death such as septicemia, mediastinitis, pneumonia and meningitis.
\end{abstract}

Methods and results: We shared the presentation of our two cases. In the first case, pharyngeal perforation and spontaneous extrusion of the $\mathrm{C} 2-\mathrm{C} 3$ fixation device occurred after eight years from surgery. The fixation device removed and the pharyngeal wall repaired surgically. In the second case, one of the upper screws perforated the posterior pharyngeal wall in the early post-operative period and migrated to the right bronchus. The fixation device excision was achieved with rigid bronchoscopy, and the pharyngeal perforation was treated symptomatically.

Conclusions: The patients who underwent anterior cervical fusion surgery that contains the $\mathrm{C} 3$ level are at risk of pharyngeal perforation. If the fixation device migrated to the thoracic cavity, it should be removed by interventional and/or surgical methods for the prevention of infection. The treatment of pharyngeal perforation after anterior cervical fusion surgery should be planned by multidisciplinary team that involves spine surgeons as well as otolaryngologists.

\section{Keywords}

Spinal fusion, Pharynx, Perforation, Respiratory aspiration, Foreign body migration, Thorax

\begin{abstract}
Abbreviations
ACSF: Anterior Cervical Spine Fusion; PEEK: Polyether Ether Ketone; CT: Computed Tomography; MRI: Magnetic Resonance Imaging
\end{abstract}

\section{Introduction}

Anterior cervical fusion surgery (ACFS) is one of the most commonly performed treatment for degenerative disc disease with or without myelopathy, trauma and neoplastic lesions of cervical spine [1]. Complications of ACFS involve dysphagia, postoperative hematoma, symptomatic recurrent laryngeal nerve palsy, Horner's syndrome and pharyngeal, hypo-pharyngeal or esophageal perforation and instrumentation pullout [2]. Pharyngeal, hypo-pharyngeal or esophageal perforations are serious complications of ACFS [3]. The perforation site is frequently the esophagus due to its anatomical location. The incidence of delayed pharyngoesophageal perforation from anterior cervical spine hardware is between $0.25 \%$ to $1.49 \%$ [4]. Another serious complication after ACFS is migrating and/or missing screws [5]. Mostly, this occurs as a result of a rupture at the pharyngeal, hypopharyngeal and/or esophageal wall. The route of the migration is the gastrointestinal trunk more frequently [6]. However, respiratory system is a very rare and alternative route for this complication, which was reported three times in the literature [7-9]. In this report, we present two complications of ACFS. The first case involves a pharyngeal perforation and spontaneous extrusion of the C2-C3 fixation device. The second case involves 

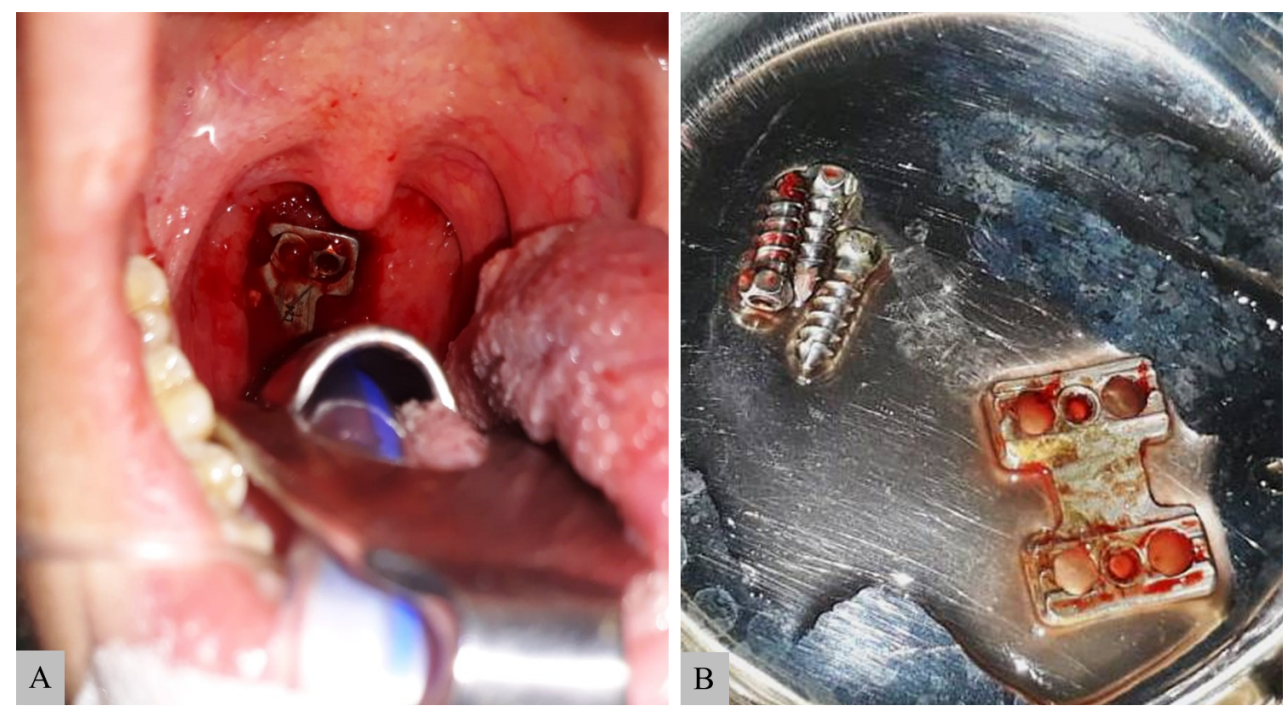

Figure 1: Preoperative view of oropharynx. A) Erosion of the oropharyngeal mucosa and the anterior cervical plates and screws are visible. No evidence of mucosal infection is observed at the borders of the perforation. Removed material; B) Of the anterior fixation device (including plate and screws) at cervical revision surgery.

the migration of the upper left screw to the thoracic cavity after anterior cervical fusion surgery at the C3-7 levels.

\section{Case Presentation}

\section{Case 1}

A 36-year-old female patient was admitted to our department with a diagnosis of traumatic listhesis at the C2-3 level after a motor vehicle accident. Neurological examination of the patient revealed no deficit. C2-3 anterior cervical discectomy, fusion with polyether ether ketone (PEEK) cage and anterior platescrew fixation surgery were performed. After eight years from surgery, the patient presented to our clinic with dysphagia. On examination, she had a sore throat for the past few weeks and had a foreign body sensation in her throat. Fiber optics pharyngoscopy was used in physical examination, and mucosal erosion was seen in the oropharyngeal region. Anterior cervical plate was visible on the posterior wall of the pharynx (Figure 1A) and there was no appearance of abscess formation or inflammation. Cervical vertebra $\mathrm{X}$-ray (Figure 2A) and CT (Figure 2B) revealed fusion at the C2-3 level, but also a perforation of the phar$\mathrm{ynx}$ wall due to the fixation device (Figure $2 \mathrm{C}$ and Figure 2D). As the patient had adequate fusion, it was concluded that there was no need for re-stabilization surgery. Therefore, surgery was planned for removal of fixation device and repair of the pharyngeal wall. During surgery, the oral cavity did not allow manipulation with a screwdriver (Figure 1B). The margins of the pharyngeal mucosa in the perforation area were seemed to be healthy (Figure $1 \mathrm{~A}$ ). So, the anterior cervical approach was applied instead of transoral approach and fixation device was removed. Broad-spectrum antibiotics and total parenteral nutrition were used in the treatment of pharynx perforation, and the wound was left to healing with secondary intention. The patient was followed up in the hospital for two weeks. During this period the symptoms of dysphagia improved, and she was discharged. In 12 weeks after discharge, the primary repair of the pharynx with removal of fixation device was failed although any infection did not occur. Since the healing was insufficient despite 12 week-period and to prevent secondary infection, the otolaryngology team has repaired the pharyngeal perforation surgically by turning the mucosal flap. The patient is currently being followed up without symptom.

\section{Case 2}

A 44-year-old male patient was admitted to the emergency department for spinal cord injury after a motor vehicle accident. His neurological examination revealed tetraplegia. Spinal radiological imaging revealed diffuse spinal cord edema (Figure $3 \mathrm{~A}$ ) and 2 $\mathrm{mm}$ epidural collection at C3-7 levels. Hemodynamic stabilization of the patient who has a spinal shock state was provided in the neuro-intensive care unit. C4-5 corpectomy and C6-7 discectomy were performed urgently to provide spinal decompression. The fusion was achieved using a distractible cage and a PEEK cage (Figure 3B). Anterior plate stabilization was performed (Figure 4A and Figure 4B). The patient's follow-up and treatment continued in the neuro-intensive care unit with the support of mechanical ventilation. Two months after the operation, a screw was detected in the right lung distal bronchus in the routine PA chest X-ray (Figure 4E). Spinal imaging revealed that the upper left screw which was fixed to the C3 corpus was not in place (Figure $4 \mathrm{C}$ and Figure 4D) and that it had migrated to the thoracic cavity after creating a perforation in the pharyngeal wall. 

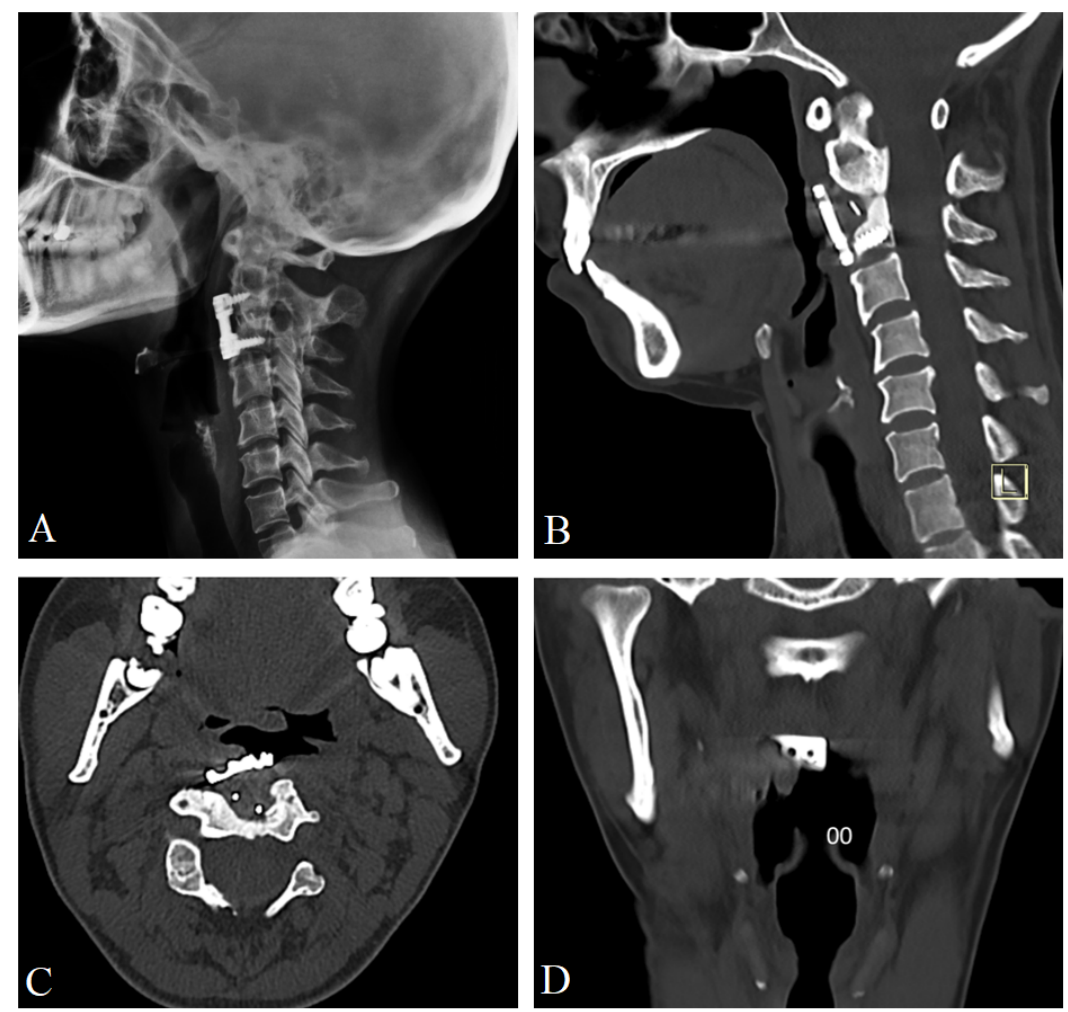

Figure 2: Lateral cervical X-ray. A) Showing adequate fusion at C2-3 level after eight years from ACSF; B) Sagittal; C) Axial; D) Coronal. Plane of cervical vertebra CT which was taken eight years after surgery, showing pharyngeal perforation and fixation device protrusion to pharyngeal cavity.
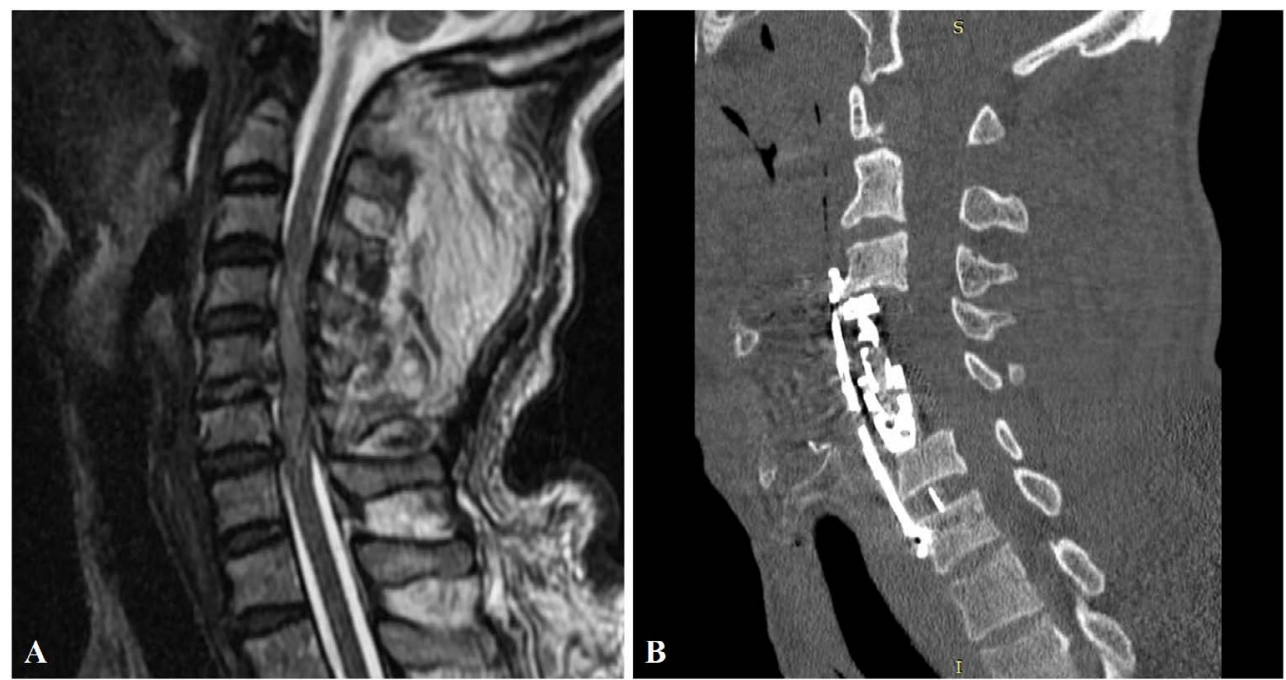

Figure 3: Preoperative MRI of the patient in the sagittal plane. A) Showing traumatic posterior C5-6 disc herniation, diffuse spinal cord edema and $2 \mathrm{~mm}$ epidural fluid collection at C3-7 levels. Postoperative cervical CT; B) Showing C4-5 corpectomies (and fusion with a distractible cage) and C5-6 discectomy (and fusion with a PEEK cage) and stabilization with anterior plate and screws.

The patient underwent rigid bronchoscopy, and the screw was removed. Upper fixation screws were revised and stabilized surgically. Since the patient had a tracheostomy and receives the enteral tube feeding, the pharyngeal perforation was left to secondary wound healing. The patient was referred to the physical therapy center after three months from the operation.

\section{Discussion and Conclusions}

After ACFS, the pharynx, hypopharynx and esophagus are at risk of perforation [10]. The reasons for perforation following cervical spine fusion are erosion of the soft tissue of the posterior pharynx and tissue ischemia due to chronic compression of the permanent fixation device [4], malpositioning of the screws in the vertebral body [11], initial inadequate positioning or engagement 

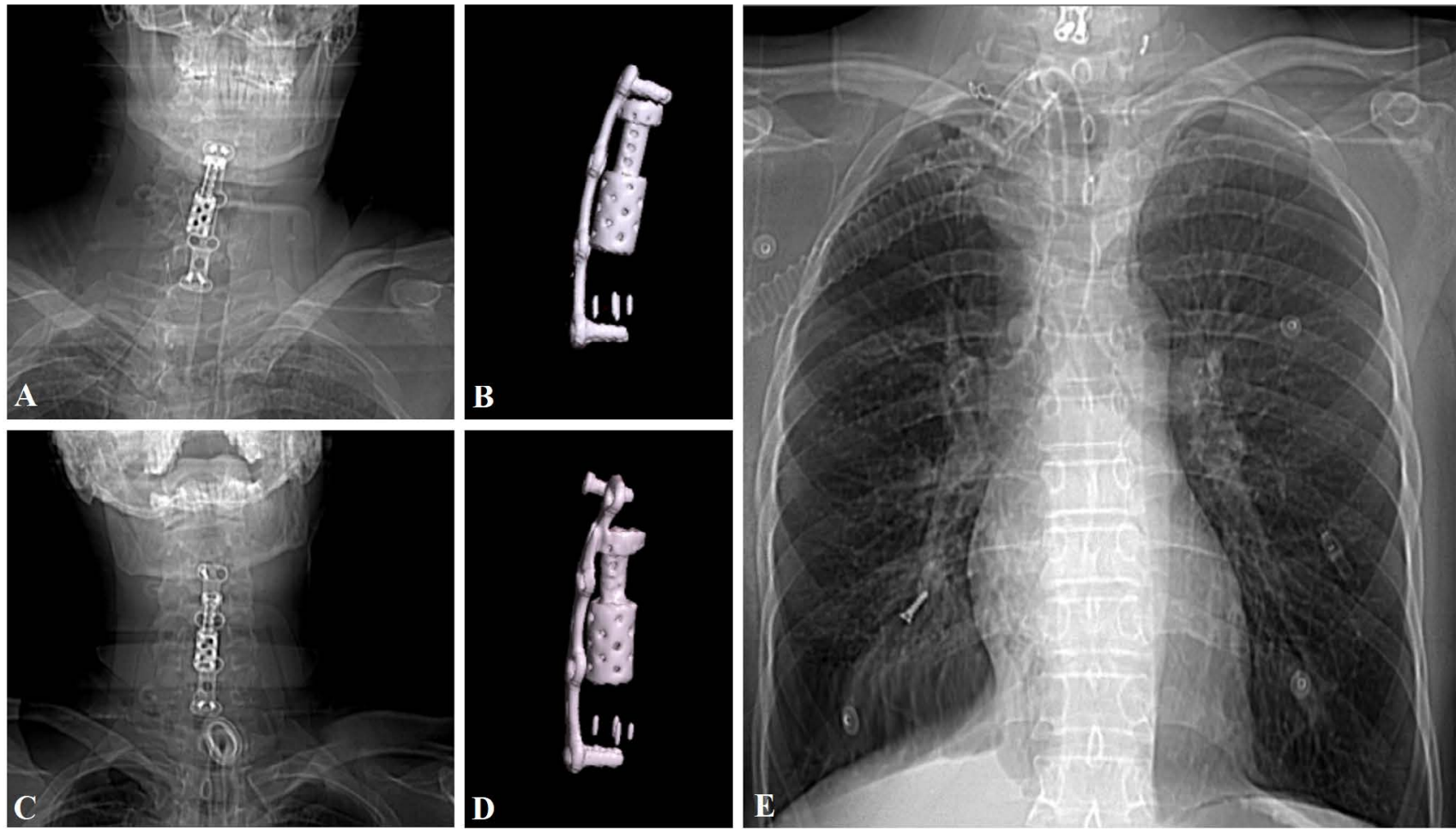

Figure 4: Early postoperative X-ray. A) And cervical CT; B) Showing that the anterior plate and screws are stable. After two months from surgery, X-ray; C) Showing that the up-left screw was lost, and cervical CT confirming that the up-left screw was migrated; D) PA Chest X-ray showing that the migrated screw was on the bronchus of the right lung (C).

of screws in a plate [6], rejection of the fixation device [12] and local infection [13].

Loosening of the screws and/or plates used in surgery and their migration may cause chronic irritation in the posterior wall of the pharyngoesophageal cavity [3]. This may cause inflammation, focal ischemia and necrosis, cellulite, formation of abscesses and perforation. In the case of perforation, the clinical picture may vary from asymptomatic to painful cervical swelling, fever, dysphagia, odynophagia, subcutaneous emphysema [14]. In our Case 1, the upper screws seem to be loosened and the plate to become forward to the pharyngeal cavity (Figure 2B). In our Case 2, one screw were seemed to loosen (Figure 4D) and the other one migrated to the right lung (Figure $4 \mathrm{E})$. Other biomechanical cause of perforation in our Case 2 could be the placement of a long construct.

According to the previous reports, to lessen the repetitive friction and microtrauma which may lead the pharyngoesophageal perforations, the anterior construct should be smooth [15]. This way will prevent the pressure necrosis. Nourbakhsh, et al. [15] recommended to remove redundant locking screws due to their potential for penetration. An exact entry point for the screws, an adequate trajectory, and cortical purchase of the screws are necessary to provide enough strength [13]. If sufficient strength is not achieved until fusion develops, the fixation device may loosen or migrate. There are two ways to com- pensate for insufficient strength. One is to change the length of the steel plate and fix the screw in a place with high-density cancellous bone. The other way is to replace with thicker, longer screws to increase the strength of fixation [16]. In patients with revision surgeries, the steel plate or screws should be changed to reinforce the fixators and avoid extrusion.

Although loosened or dislodged fixation devices are predictable causes for perforation, it was reported that the fixation devices were seemed to be intact intraoperatively in some cases in the literature [4].

Despite the mechanism of formation in both pharyngeal perforations and esophageal perforations is similar, pharyngeal perforations are much rarer. This may also be due to the level of the surgical field. Because the oropharynx is the area behind the oral cavity extending from the uvula to the level of the hyoid bone [17]. Hyoid bone is at the third cervical vertebra (C3) level at rest. In the literature, all of the patients who has pharyngeal perforation after ACFS, the initial operation involves the level of C3 [18-20]. In the present case, the fixation devices of both patients caused pharyngeal perforation at the $\mathrm{C} 3$ level. In the second case, in addition to the pharyngeal perforation, one of the fixation screws migrated to the right bronchus.

The clinical presentation of patients varies depending on the neurological state and whether there is an infection. The complaints of the cases of pharyngeal perforation in the literature vary from progressive difficulty 
to swallowing, painful swallowing or sore throat, weight loss, foreign body sensation in the throat and coughing out to the fixation device [18-20]. If the perforation is not diagnosed in the early period and the treatment is delayed, catastrophic consequences may occur which may cause death such as septicemia, mediastinitis, pneumonia and meningitis [10].

In the literature, the vast majority of cases [18-20] were treated by removal of the remaining and/or migrated fixation device and secondary wound healing. Rama-Lopez, et al. [19] reported a case of ulcerated pharyngeal perforation and treated with surgical debridement.

Our Case 1 was re-operated 12 weeks after the second surgery due to insufficient mucosal healing, despite the infectious status did not occur. Although many cases [18-20] are treated with broad-spectrum antibiotics and secondary wound healing at early stages some infected perforations require surgical drainage, mucosal or muscle flap, and revision surgeries [3]. Phommachanh, et al. [4] reported five patients with pharyngoesophageal perforation. Four patients managed with primary closure of the defect with a pedicled sternocleidomastoid muscle. One patient was treated with a pectoralis muscle flap.

There are several reports $[2,21,22]$ referring to partial or total migration of the fixation device to gastrointestinal system via oral cavity and pharyngoesophageal perforation after ACFS. Rare cases such as aspiration and intrathoracic migration have also been reported in the literature [7-9]. The clinical presentation of foreign bodies migrating to the thorax involves acute airway obstruction and recurrent infections [9]. In the literature, one of the patients [8] had intermittent fever, and the other one [9] had coughing for 2 months. In our second case, the patient had no complaints because of no verbal response due to tracheostomy and no fever or coughing was found. In order to prevent infections, all migrated foreign bodies should be removed by interventional and/or surgical methods. In the literature, wedge resection was performed in two patients [8,9] due to severe adhesion and granulation. In the other patient [7], the migrated screw was removed with rigid bronchoscope as applied in our second case.

Pharyngeal perforations and fixation device migrations are rare but serious complications of ACFS. They may occur during the surgery, in the early or late postoperative period. Our experience indicates that if the patients have complaints such as swallowing difficulties and sore throat after ACFS, these should not be ignored, and radiological examination should be performed. Surgery for revision should be scheduled as soon as possible when fixation device loosening or migration appears in the neck or thorac- ic region, to prevent potentially fatal complications. Multidisciplinary treatment including comprehensive physical examination with otolaryngologists, infection prophylaxis using broad-spectrum antibiotics, parenteral or enteral nutrition and surgical repair in complicated cases is essential for successful treatment of pharyngeal perforations.

\section{Declarations}

All procedures performed in studies involving human participants were in accordance with the ethical standards of the institutional and/or national research committee (Gazi University Ethics Committee) and with the 1964 Helsinki Declaration and its later amendments or comparable ethical standards. The patients have consented to the submission of the case reports for submission to the journal. All data generated or analyzed during this study are included in this published article. The authors have no conflict of interest to declare. We declare that we have no known competing financial interests or personal relationships that could have appeared to influence the work reported in this paper.

\section{Disclosure}

We declare that we have no known competing financial interests or personal relationships that could have appeared to influence the work reported in this paper.

All authors have contributed significantly to the work, have read the manuscript, attest to the validity and legitimacy of the data and its interpretation, and agree to its submission to the Neurosurgery Cases and Reviews.

\section{References}

1. Anandaswamy T, Pujari V, Shivanna S, Manjunath A (2012) Delayed pharyngoesophageal perforation following anterior cervical spine surgery: An incidental finding. J Anaesthesiol Clin Pharmacol 28: 139.

2. Cagli S, Isik HS, Zileli M (2009) Cervical screw missing secondary to delayed esophageal fistula: Case report. Turk Neurosurg 19: 437-440.

3. Dakwar E, Uribe JS, Padhya TA, Vale FL (2009) Management of delayed esophageal perforations after anterior cervical spinal surgery. J Neurosurg Spine 11: 320-325.

4. Demirkaya A, Ersen E, Kılıc B (2016) Wandering cervical screw: From cervical vertebra to intermediate bronchus. Asian Cardiovasc Thorac Ann 24: 827-828.

5. Fountas KN, Kapsalaki EZ, Nikolakakos LG, Smisson HF, Johnston KW, et al. (2007) Anterior cervical discectomy and fusion associated complications. Spine (Phila Pa 1976) 32: $2310-2317$.

6. Fujibayashi S, Shikata J, Kamiya N, Tanaka C (2000) Missing anterior cervical plate and screws: A case report. Spine (Phila Pa 1976) 25: 2258-2261.

7. Gazzeri R, Tamorri M, Faiola A, Gazzeri G (2008) Delayed migration of a screw into the gastrointestinal tract after anterior cervical spine plating. Spine (Phila Pa 1976) 33: E268-E271. 
8. Geyer TE, Foy MA (2001) Oral extrusion of a screw after anterior cervical spine plating. Spine (Phila Pa 1976) 26: 1814-1816.

9. Hung C-C, Guo J-H, Cheng Y-K, Cho D-Y (2016) Delayed anterior cervical screws migrating simultaneously to the lung and stomach. Spine J 16: e263-e264.

10. Kalaycioglu S, Fındık G, Aydogdu K, Dagli G, Kaya S (2010) Intrathoracic migration of a screw in the cervical spine: A case report. Turkish J Thorac Cardiovasc Surg 18: 338-340.

11. Kapu R, Pande A, Ramamurthi R, Singh M, Vasudevan M (2012) Delayed anterior cervical plate dislodgement with pharyngeal wall perforation and oral extrusion of cervical plate screw after 8 years: A very rare complication. J Craniovertebr Junction Spine 3: 19.

12. Kelly MF, Spiegel J, Rizzo KA, Zwillenberg D (1991) Delayed pharyngoesophageal perforation: A complication of anterior spine surgery. Ann Otol Rhinol Laryngol 100: 201205.

13. Lee EJ, Jang JW, Choi SH, Rhim SC (2012) Delayed pharyngeal extrusion of an anterior odontoid screw. Korean J Spine 9: 289.

14. Li Y, Zhu Q-S, Liu J-C, Wu Y-T (2015) Acute cervical epidural hematoma, screw pullout, and esophageal perforation after anterior cervical corpectomy surgery: report of a case. Int Surg 100: 334-340.

15. Nourbakhsh A, Garges KJ (2007) Esophageal perforation with a locking screw. Spine (Phila Pa 1976) 32: E428-E435.
16. Park S-A, Lee J-H, Nam Y-S, An X, Han S-H, et al. (2013) Topographical anatomy of the anterior cervical approach for c2-3 level. Eur Spine J 22: 1497-1503.

17. Phommachanh V, Patil YJ, McCaffrey TV, Vale F, Freeman TB, et al. (2010) Otolaryngologic management of delayed pharyngoesophageal perforation following anterior cervical spine surgery. Laryngoscope NA-NA.

18. Quadri SA, Capua J, Ramakrishnan V, Sweiss R, Cabanne $\mathrm{M}$, et al. (2017) A rare case of pharyngeal perforation and expectoration of an entire anterior cervical fixation construct. J Neurosurg Spine 26: 560-566.

19. Rama-Lopez J, Tomas-Fernandez M, Garcia-Garza C, Martinez-Madrigal M (2018) Pharyngeal perforation after anterior cervical spine surgery treated by transoral endoscopic surgery. Head Neck 40: E13-E16.

20. Sharma RR, Sethu AU, Lad SD, Turel KE, Pawar SJ (2001) Pharyngeal perforation and spontaneous extrusion of the cervical graft with its fixation device: A late complication of C2-C3 fusion via anterior approach. J Clin Neurosci 8: 464468.

21. Sun L, Song Y, Liu L, Gong Q, Liu H, et al. (2012) Causes, treatment and prevention of esophageal fistulas in anterior cervical spine surgery. Orthop Surg 4: 241-246.

22. Whitecloud TS (1988) Anterior surgery for cervical spondylotic myelopathy. Smith-Robinson, Cloward, and vertebrectomy. Spine (Phila Pa 1976) 13: 861-863. 